\title{
Labyrinthe
}

$30 \mid 2008$ (2)

Ecologie $=X$

\section{L'écocritique et la science : vers une consilience?}

\section{Glen Love}

Traducteur : Laurent Ferri

\section{OpenEdition}

Journals

Édition électronique

URL : https://journals.openedition.org/labyrinthe/3764

DOI : 10.4000/labyrinthe.3764

ISSN : 1950-6031

Éditeur

Hermann

Édition imprimée

Date de publication : 10 juin 2008

Pagination : 17-36

ISBN : 978-2-9526131-7-0

Référence électronique

Glen Love, «L'écocritique et la science : vers une consilience? », Labyrinthe [En ligne], 30 | 2008 (2), mis en ligne le 27 mai 2008, consulté le 12 janvier 2022. URL : http://journals.openedition.org/labyrinthe/ 3764 ; DOI : https://doi.org/10.4000/labyrinthe.3764

Propriété intellectuelle 


\section{L'ÉCOCRITIQUE ET LA SCIENCE: VERS UNE CONSILIENCE ?*}

Glen Love

Quelles que soient les difficultés, et les risques de manipulation maladroite, l'interdisciplinarité semble être la seule méthode rationnelle permettant de réconcilier deux cultures que tout sépare: on le sait depuis les travaux de C.P. Snow, qui popularisa il y a quarante ans la notion de "fossé» entre les sciences et les humanités. Selon Joseph Meeker, le développement de l'écocritique (dont il est un des inventeurs), est lié à la dynamique interdisciplinaire dans l'enseignement: «La vogue croissante de l'interdisciplinarité dans les universités n'est pas une lubie d'universitaire; c'est une manière de répondre à un besoin que les gens, partout, ressentent de plus en plus: besoin de trouver un sens à leur existence, et de comprendre le monde qui les entoure'.» L'étude conjointe de la littérature et de l'environnement s'est imposée en réponse à ces préoccupations.

L'écocritique, nouveau champ des études littéraires, semble particulièrement bien placée pour tirer parti des croisements interdisciplinaires avec les sciences, en rupture avec l'opposition conflictuelle entre les deux cultures. Il apparaît clairement aujourd'hui que le refus en bloc de la science n'est pas plus défendable au plan intellectuel qu'il n'est efficace au plan politique. Défendre la science ne signifie pas valider ses excès, ni absoudre les péchés dont nous sommes aujourd'hui les témoins - par exemple, avec les phénomènes de «fuite en avant" technologique. Cela veut dire qu'en matière de recherche, la méthode scientifique reste le meilleur moyen dont nous disposons pour comprendre notre monde, et pour tenter de trouver des solutions aux problèmes de pollution, de surpopulation, de spoliation des ressources naturelles. De ce point de vue, l'essor de l'écocritique est un aspect de

\footnotetext{
* Texte paru à l'origine en anglais sous le titre « Ecocriticism and Science: Toward Consilience? ", New Literary History, vol. 30, $\mathrm{n}^{\circ}$ 3, été 1999, p. 561-576. O 1999 Glen Love.

1. Joseph W. Meeker, «Fields of Danger and the Wilderness of Wisdom », North American Review, vol. 263, mars 1978, p. 71.
} 
la sensibilisation croissante, à l'échelle mondiale, aux problèmes d'environnement.

Faire de l'écocritique, c'est répondre à l'appel de l'interdisciplinarité scientifique. La littérature est par nature une activité qui nous met en situation de dépendance mutuelle; pareillement, la prise de conscience environnementale renforce notre sens d'une interdépendance, en l'élargissant à des contextes qui ne sont plus strictement interhumains. Penser la littérature sur un mode écologique nous oblige donc à considérer le monde non humain avec le même sérieux que les autres méthodes critiques ont déployé dans l'étude de la société et de la culture humaines. Ceci, me semble-t-il, est le principal défi pour l'écocritique; c'est aussi sa plus grande chance.

Prendre le monde au sérieux signifie, entre autres choses, apprendre quelque chose de scientifique à son sujet. Richard Levin, qui a finement analysé les bévues interdisciplinaires commises par des littéraires, a formulé plusieurs propositions pour éviter de telles erreurs: il propose par exemple de recourir à l'expertise de relecteurs issus de différentes disciplines quand il s'agit de livres ou d'articles qui prétendent être interdisciplinaires; je pense pour ma part qu'il faudrait aussi permettre aux étudiants, en premier comme en deuxième cycle, d'avoir une double major; ou encore redoubler d'efforts contre la politisation excessive à l'intérieur des disciplines. D'autres stratégies utiles pourraient, de mon point de vue, comprendre la lecture régulière d'un hebdomadaire pluridisciplinaire comme Science, dont un tiers du contenu est toujours destiné à des non-spécialistes. L'étudiant en quête de transdisciplinarité pourrait assister à des conférences publiques, participer à des groupes de discussion (même dans les cas où il s'attend à être en désaccord avec les conclusions), faire la connaissance d'un « vrai» scientifique, et visiter un laboratoire. En fin de compte, le plus important, comme dit Levin, « est d'en savoir assez pour que les spécialistes d'en face ne trouvent pas absurde ma façon d'utiliser leur discipline dans ma recherche ${ }^{2}$ ».

Il va de soi que le recours à des témoignages d'experts du domaine exploré sera nécessaire, mais cela ne veut pas dire que l'auteur sera nié, gommé, au terme de cette immersion. Comme l'a dit Gaston Bachelard:

2. Richard Levin, $\alpha$ The New Interdisciplinarity in Literary Criticism», dans Nancy Easterlin et Barbara Riebling (dir.), After Poststructuralism, Evanston, Northwestern University Press, 1993, p. 33-34. 


\title{
L'écocritique et la science: vers une consilience?
}

«La science moderne prend I'homme dans un monde nouveau. Si I'homme pense la science, il est renouvelé comme être pensant. II adhère à une indéniable hiérarchie de la pensée ${ }^{3}$. " Mary McAllester Jones, qui a traduit et commenté le philosophe, note à son propos:

\begin{abstract}
Bachelard estime que chacun d'entre nous peut entrer dans la science par le biais de la lecture... Le fait de ne pas comprendre est sans importance; ce qui est important, c'est le travail de l'esprit quand il est confronté au réseau de références qui sous-tend l'argument scientifique. Lire la production scientifique moderne est très difficile, mais c'est un travail productif, car il nous permet, plus que notre vie ordinaire, de faire l'expérience de la cohérence organisée; parce que les données sont constamment rectifiées, tout est constamment réordonné selon la logique d'ordre possible, de discontinuité maintenant le lien entre les parties, de différence encadrée par un système $[G B$, p. 174].
\end{abstract}

L'humaniste trouvera un motif littéraire parallèle dans des œuvres aussi connues que Moby Dick de Melville et Les Raisons de la colère de Steinbeck. Y alternent et s'entrelacent des chapitres scientifiques et des passages narratifs qui mettent surtout en valeur l'humanité des personnages. Melville, par exemple, n'accable son lecteur de connaissances en sciences naturelles (que n'apprend-t-on pas sur les cétacés!) que pour mettre en doute la capacité de la science - et de l'esprit humain en général - à comprendre la dynamique illimitée de la nature. L'expérience salutaire que nous faisons à la lecture du livre est celle d'un système de contrepoids, comme dans le puissant mouvement oscillatoire décrit par Bachelard. En ces occasions toutes simples, l'interconnexion subtile entre la science et l'art nous ouvre la possibilité gratifiante d'une unité retrouvée. On se souvient de la "prière du lecteur dévorant" chez Bachelard: «Donne-nous aujourd'hui notre faim [de livres] quotidienne" [cité dans $G B$, p. 176].

Les tonnes d'informations livrées par Melville sur les baleines, tout comme la vision écologique de Steinbeck quand il nous raconte l'odyssée de Joad, donnent raison au critique C.P. Snow. Dans un essai de 1964, qui reprenait en fait sa conférence de 1959 sur les «deux cultures» (voir plus haut), Snow estimait que la biologie est celle qui,

3. Cité dans Mary McAllester Jones, Gaston Bachelard, Subversive Humanist, Madison, The University of Wisconsin Press, 1991, p. 174 [désormais noté GB]. 
de toutes les sciences dites naturelles, nous permet le mieux, à nous les humanists qui nous efforçons de travailler vraiment de manière pluridisciplinaire, de traiter sérieusement de la nature. Dans le livre qu'il publia peu de temps avant sa mort, Aldous Huxley disait la même chose: "Il est évident que la biologie nous parle davantage, du point de vue de l'expérience humaine, que les sciences exactes comme la physique et la chimie». Huxley presse les humanists de révéler aux hommes des interconnexions qu'ils sont les seuls à voir, parce qu'ils "perçoivent et ressentent avec leur cœur, et survolent pour ainsi dire les savoirs scientifiques ${ }^{4} »$. S'il est vrai que le propre de l'homme est de se prendre luimême comme premier objet d'étude, dit Huxley après Pope, la Nature vient immédiatement en second, et les liens qui unissent l'homme et la nature (ce dont l'écologie se préoccupe) sont de la plus haute importance:

À la lumière de ce que nous savons aujourd'hui des relations entre les êtres vivants et avec leur environnement inorganique - et au vu de ce que, à nos dépens, nous apprenons au sujet de la surpopulation, de notre ruineuse agriculture, de la déforestation et de la destruction insensée des pâturages, de la pollution de l'eau et de l'air, de la stérilisation ou de la disparition complète de sols autrefois fertiles, il est maintenant devenu tout à fait clair que la même règle d'or s'applique non seulement aux relations entre individus et entre sociétés humaines, mais aussi aux relations avec les autres créatures vivantes, et avec la planète sur laquelle nous voyageons à travers l'espace et le temps [LS, p. 108-109].

Non seulement Huxley, de manière prémonitoire, alertait ses contemporains au sujet de l'environnement, mais il appelait les communautés scientifique et littéraire à se rapprocher, à approfondir leur relation pour découvrir ensemble «la matière première d'une nouvelle façon d'écrire sur la nature» $[L S$, p. 110] (et - sans doute en aurait-il été d'accord - une nouvelle façon de faire de la critique littéraire).

La sombre prophétie de Huxley fut certainement influencée par le best-seller de Rachel Carson, Silent Spring (1962). Comme le souligne Lawrence Buell, l'intérêt du livre s'explique autant par les qualités d'invention de l'auteur, et par ses talents de conteur, que par le sérieux de la thèse

4. Aldous Huxley, Literature and Science, New York, Harper and Row, 1963, p. 79 [dorénavant noté $L S$ ]. L'essai écrit par Snow en 1964, «The Two Cultures: A Second Look» a été rédité dans C.P. Snow, The Two Cultures, Cambridge, Cambridge University Press, 1993. 
scientifique 5 . Biologiste de profession, mais animée de son ambition de faire véritablement œuvre d'écrivain, Carson a de fortes affinités avec Aldous Huxley du point de vue du rapprochement littérature/biologie. En effet, on peut considérer Silent Spring comme la version écologique de Brave New World: le sujet est le même, à savoir la raréfaction de la vie sur terre. En ce qu'il marquait l'irruption de l'écologisme radical, le livre de Carson eut un énorme impact: tout d'un coup, le grand public, en même temps qu'il était éduqué scientifiquement à la biologie, prenait conscience des possibilités de la catastrophe écologique.

L'écocritique prit racine dans cette prise de conscience, et se développa durant le reste des années 1960 et 1970. Je fis moi-même paraître un essai d'écocritique, «L'écologie en Arcadie», qui étudiait les représentations de la dégradation de l'environnement dans la littérature contemporaine et le cinéma ${ }^{6}$. Je me souviens que le sujet était alors considéré, quand il l'était, comme tout à fait marginal au sein des études littéraires. L'ouvrage, désormais classique, de Leo Marx, The Machine in the Garden (1964), fut certes pour le jeune chercheur que j'étais, travaillant sur la littérature américaine dans une perspective environnementaliste, une source d'inspiration formidable. Toutefois, dans les dernières pages de son livre, Marx décrétait que le souci de protéger la nature contre sa dégradation appartenait désormais au passé, la fin du $\mathrm{XIX}^{\mathrm{e}}$ siècle ayant vu en Amérique le triomphe définitif des machines sur la vie aux champs. J'observais, bien au contraire, que le combat continuait autour de moi, plus féroce que jamais. Tout le monde, $y$ compris Marx quand il se repencha sur le sujet vingt ans plus tard, devait en convenir - même s'il refusa toujours de voir dans l'écologie politique autre chose qu'une option idéologique parmi d'autres?.

La connexion entre la littérature et la biologie trouva sa première grande expression critique avec la publication en 1974 du livre de Joseph W. Meeker, The Comedy of Survival: Literary Studies in Ecology.

5. Lawrence Buell, The Environmental Imagination, Cambridge, Cambridge University Press, 1995, p. 290-293.

6. Glen A. Love, « Ecology in Arcadia », The Colorado Quarterly, vol. 21, Automne 1972, p. 175-85. Un autre ouvrage datant de cette époque (Glen A. Love and Rhoda M. Love (dir.), Ecological Crisis, New York, Harcourt, Grace, 1970), publié conjointement par un chercheur en sciences humaines et une biologiste, fut le premier à décrypter le mouvement environnementaliste.

7. Leo Marx, « Pastoralism in America », dans Sacvan Berkovitch and Myra Jehlen (dir.), Ideology and Classic American Literature, Cambridge, Cambridge University Press, 1986, p. 36-69. 
Rétrospectivement, il m'apparaît comme l'un des livres de critique littéraire les plus importants des vingt-cinq dernières années. C'est aussi, malheureusement, un des moins lus. Meeker était à la fois titulaire de diplômes supérieurs en écologie et en éthologie, l'auteur de manuels de biologie pour le premier cycle universitaire, et docteur en littérature comparée. Il est le premier à avoir étudié en profondeur la littérature du point de vue écologique, et plus précisément dans une perspective évolutionniste. En bon comparatiste, Meeker part de l'analogie selon laquelle la «littérature comparée est aux humanités ce que l'écologie est aux sciences naturelles » pour faire valoir le caractère inséparable de la littérature et de l'environnement (qui englobe «la nature»):

Si l'on pose que l'invention de la littérature est une importante caractéristique de l'espèce humaine, il convient d'examiner attentivement et honnêtement quelle influence elle a effectivement sur le comportement humain et sur l'environnement - quel rôle elle joue dans le bien-être et la survie de l'humanité, dans les relations qu'entretient l'homme avec les autres espèces et avec le monde qui nous entoure. S'agit-il d'une activité qui nous permet de mieux nous adapter au monde, ou bien au contraire qui nous en éloigne? Du point de vue de l'évolution et de la sélection naturelle des espèces, la littérature favorise-t-elle plutôt notre survie, ou notre extinction ${ }^{8}$ ?

Le livre de Meeker faillit obtenir le prix Pulitzer, finalement attribué à Annie Dillard pour Pilgrim at Tinker Creek: certes un autre très bon livre sur les relations de l'homme avec la nature, mais aussi une œuvre de littérature sur la nature, relevant clairement du genre nature writing, contrairement au livre de Meeker qui déconcerta parce qu'il échappait à toutes les classifications. Le travail de Meeker était par nature interdisciplinaire, à la différence des milieux universitaires et des critiques, très spécialisés: Meeker savait parfaitement que son œuvre, en avance sur son temps, passerait largement inaperçue. Cependant, une nouvelle édition est parue en 1997 à la faveur du succès croissant de l'écocritique $^{9}$.

Je crois pouvoir dire que tout un segment de la réflexion sur le vivant s'est développé en conjonction étroite avec l'écocritique, et plus généralement l'étude de la littérature en rapport avec l'environnement.

8. Joseph W. Meeker, The Comedy of Survival, New York, 1974, p. 11, 3-4.

9. Tucson, Arizona University Press, 1997. 
Grâce à Silent Spring de Carson, qui apportait des preuves irréfutables de la destruction de l'environnement, une sous-discipline assez obscure de la biologie, l'écologie, est sortie des laboratoires pour devenir un thème de débat public; il a eu l'effet d'un catalyseur de la pensée novatrice, interdisciplinaire, dans l'université. Il était logique que la biologie devînt le point de connexion entre littérature et sciences de la nature, étant la plus accessible de toutes. De plus, comme l'a souligné Huxley, les biologistes peuvent se targuer de ne jamais perdre de vue la vie humaine.

Toutefois, entre Meeker et les années 1990, la plupart des chercheurs en littérature et sciences humaines qui transgressaient les disciplines ont regardé du côté de théories issues de la physique, comme le chaos et l'incertitude, plutôt que du côté des sciences du vivant; en particulier, tout ce qui se rapporte aux théories de l'évolution s'est trouvé délaissé. Il est possible qu'ils aient pris peur après les réactions suscitées par le livre d'Edward O. Wilson, Sociobiology: The New Synthesis (1975) - épisode sur lequel je reviendrai plus loin. Mais tout cela a changé au cours de la présente décennie, quand un certain nombre d'ouvrages très bien documentés ont été publiés sur la littérature et l'environnement, et dans bien des cas, sur la question spécifique de l'évolution. On peut citer Romantic Ecology de Jonathan Bate (1991), A Blessed Rage of Order d'Alexander J. Argyros (1991), Ecological Literary Criticism de Karl Kroeber (1994), The Environmental Imagination de Lawrence Buell (1995), Evolution and Literary Theory de Joseph Carroll (1995), et Mimesis and the Human Animal de Robert Storey (1996). Un certain nombre de textes publiés dans l'anthologie critique de Cheryll Glotfelty et Harold Fromm, The Ecocriticism Reader (1996), s'inscrivent également dans ce contexte.

Parmi tous ces livres, celui de Joseph Carroll constitue la tentative la plus ambitieuse à ce jour d'appliquer les idées d'évolution biologique à la construction d'une théorie littéraire d'ensemble. Il avance un formidable argument pour démanteler le poststructuralisme, qui selon lui repose sur des principes malsains, parce qu'il s'oppose à «toute la structure de la connaissance scientifique, en particulier la connaissance biologique $^{10}$.» Son argument contre le paradigme poststructuraliste est

10. Joseph Carroll, Evolution and Literary Theory, Columbia, University of Missouri Press, 1995, p. 54 [dorénavant noté $E L T$ ]. 
résumé dans des termes familiers aux adeptes de l'écocritique: "Le monde enrichi de l'expérience acquise au sein de la réalité a été vidé de sa substance, et on nous offre à la place de faire joujou et de nous exciter avec l'autoréférentialité du langage. Cette vision de la vie et de la littérature est sèche et appauvrie, toute gratuite et fausse. Elle ne fait que dépeupler le monde, et pour arriver à cette fin, rend compte de manière erronée de notre expérience dans le monde» [ELT, p. 466].

Par quoi remplacer le poststructuralisme? Carroll soutient que, de toutes les façons d'expliquer sérieusement la vie, en particulier la vie humaine, la théorie évolutionniste est la plus adéquate et la plus complète: «Par voie de conséquence, elle nous fournit une base adéquate pour tout travail sérieux sur la culture et la littérature» [ELT, p. 467].

Une des choses les plus intéressantes dans le livre de Carroll est qu'il répond implicitement à ceux qui accusent l'écocritique de n'avoir - à l'instar de sa discipline alliée, l'écologie - aucun fondement théorique qui soit accepté par tous ${ }^{11}$. En fait, la théorie unifiante, dans l'étude de Carroll comme dans l'écologie, est l'évolutionnisme biologique.

Carroll conclut son livre sur une note à la fois confiante et mélancolique: d'un côté, il ne doute pas que le paradigme évolutionniste finira par s'imposer dans les sciences sociales d'ici à vingt ans, du fait qu'il permet d'expliquer tant de choses; de l'autre, il se résigne à ce que les départements de littérature ne lui réservent pas bon accueil avant longtemps. Quand il a rendu compte du livre de Carroll, Robert Storey, adepte du même paradigme, a dit son enthousiasme; mais il a également prédit qu'il serait mal reçu par les départements de littérature, hautement politisés. Il citait comme précédent la réception du livre d'Edward O. Wilson, Sociobiology: The New Synthesis, dont le dernier chapitre consacré à l'influence de la génétique sur les comportements humains s'était heurté à la résistance farouche des marxistes, entre autres $^{12}$.

Les travaux de Wilson méritent que l'on s'y arrête. Chercheur et théoricien de l'évolutionnisme biologique de rang mondial, Wilson est par ailleurs un écrivain exceptionnellement doué: c'est pourquoi je

11. Cette objection a été soulevée dans John P. O'Grady's «Unhandling our Perspective», ISLE, vol.2, printemps 1994, p. 117-21.

12. Robert Storey, note de lecture sur Evolution and Literary Theory, dans Modern Philology, $\mathrm{n}^{\circ} 94$, 1997, p. 350-354. 
considère ses travaux comme parmi les plus importants et les plus stimulants qui soient en matière de recherche interdisciplinaire et d'écocritique. Deux de ses livres, On Human Nature (1978) et The Ants (1990, avec Bert Hölldobler), ont remporté le prix Pulitzer; il a reçu par ailleurs de nombreuses récompenses aux États-Unis et dans le monde pour ses travaux scientifiques. Enfin, Wilson est un des leaders du mouvement écologique aux États-Unis: il est connu pour sa défense éloquente de la biodiversité, élément nécessaire à la continuation du monde tel que nous le connaissons. Cet aspect de sa carrière est retracé dans La Diversité de la vie (1992). Sa Biophilia (1984), un plaidoyer pour l'affinité naturelle de l'humanité avec d'autres formes de vie, fut suivi d'un ouvrage collectif, The Biophilia Hypothesis (1993), codirigé avec Stephen R. Kellert. Wilson y présente sa théorie, la biophilie, et la soumet a l'examen d'un groupe d'universitaires venus de plusieurs disciplines. La somme de leurs essais compose le volume. Bien qu'ils n'arrivent à aucune conclusion définitive sur cette question vaste et complexe, leurs commentaires sont extraordinairement intéressants et importants. En tout cas, la démarche de Wilson, consistant à soumettre son hypothèse à l'évaluation d'experts venus d'autres disciplines, est exemplaire du point de vue de la méthode scientifique. Les chercheurs interdisciplinaires à l'extérieur des sciences dures feraient bien de s'en inspirer.

Dans le même temps, l'attention que Wilson porte aux œuvres littéraires, à la tradition humaniste, et à l'histoire des idées - dans Biophilia, mais aussi dans son autobiographie, Naturalist (1994), ou encore dans son récent Consilience (1998) - font de lui une figure importante dans les humanités. On est en droit de réclamer pour Wilson l'attention des littéraires: s'il est un écrivain vraiment unique en son genre, et qui a trouvé son public, c'est que ses réflexions philosophiques tout à fait personnelles s'allient à une très vaste culture scientifique. Du coup, ses écrits sur la nature alternent l'observation et la réflexion - ce qui est un mouvement structurel sous-jacent à tout travail dans ce domaine; avec Wilson, nous faisons comme rarement l'expérience de ce que Bachelard appelle la lecture scientifique, ou l'esprit réordonne et ressaisit en permanence la pensée ordonnée qui lui est soumise.

Avec Storey et d'autres, je déplore que Wilson reste si peu connu, ou quand il l'est, diabolisé, au sein des humanités. Les mêmes idées ont 
été rapidement adoptées dans d'autres domaines, notamment les sciences sociales ${ }^{13}$. Selon Robert Wright, «le succès de la sociobiologie est universel, même si on n'ose pas prononcer le $\operatorname{mot}^{14}$ ». Paradoxalement, c'est ce mot auquel est associé, à tort ou à raison, le nom de Wilson. L'historien Carl Degler conclut son ouvrage In Search of Human Nature, par cette observation: bien qu'elle ait rompu avec le darwinisme social qui la caractérisait autrefois, la biologie doit encore faire face à une opposition considérable ${ }^{15}$.

Le dernier livre de Wilson, Consilience: The Unity of Knowledge, est à ce jour le plus ambitieux en matière de synthèse programmée entre les disciplines; on peut dire qu'il y pousse l'interdisciplinarité à ses limites. Le titre de cet ouvrage, impressionnant dans ses visées comme dans son encyclopédisme, renvoie à une tentative de synthèse de toutes les sciences opérée, en 1840, par William Whewell. Pour Wilson, la convergence dans les sciences s'explique par les chaînes d'explication causale, qui transgressent l'ensemble des frontières disciplinaires ${ }^{16}$. Cette théorie se trouve vérifiée dans le domaine des sciences naturelles, où les frontières disciplinaires ont disparu ou sont en voie de disparition, mais aussi dans la chimie, la physique, ou au sein de la biologie - où les sous-domaines disciplinaires comme la biophysique, la biochimie, la biogénétique et la chimie organique convergent au point de se confondre. Il écrit: «Il n'y a jamais eu de moment plus opportun pour la collaboration entre scientifiques et philosophes; ils peuvent en particulier se retrouver à la frontière entre la biologie, les sciences sociales, et les humanités. Nous approchons d'une nouvelle ère de synthèse, et l'effort de conciliation est le plus grand de tous les défis intellectuels. "

Wilson reconnaît que cette affirmation va en inquiéter plus d'un, et il anticipe par avance la liste de reproches qui lui seront faits: "Confusion des genres, simplisme, réductionnisme ontologique, scientisme, et d'autres péchés rendus odieux au public par le suffixe -isme.

13. Dans ce contexte, voir par exemple Mary Maxwell (dir.), The Sociobiological Imagination, Albany, Suny Press, 1991.

14. Robert Wright, Three Scientists and Their Gods, New York, 1988, p. 180.

15. Carl Degler, In Search of Human Nature, New York, 1991.

16. Edward O. Wilson, Consilience: The Unity of Knowledge, New York, Oxford University Press, 1998, p. 8 [dorénavant noté $C$ ]. 


\section{L'écocritique et la science: vers une consilience?}

Ce à quoi je réponds en plaidant coupable, coupable, et encore coupable» $[C, \mathrm{p}$. 11]. Il pousse plus loin son argument en faisant valoir que le réductionnisme scientifique, un corollaire de la convergence des sciences, est par exemple la raison du succès des sciences naturelles. II postule que l'étude de la culture, en supposant que la convergence est bien la direction que prend la recherche, va se répartir in fine et pour l'essentiel entre deux grands domaines: «Les sciences naturelles et les humanités seront les deux branches de tout apprentissage au $\mathrm{XxI}^{\mathrm{e}}$ siècle. Les sciences sociales vont continuer de faire scission (un processus douloureux mais déjà bien engagé), pour rejoindre l'une ou l'autre; certaines s'inscriront au sein ou dans la continuité de la biologie, d'autres fusionneront avec les humanités. Ces disciplines continueront à exister mais sous une forme radicalement différente. Ce faisant, les humanités $[\ldots]$ vont se rapprocher des sciences et même pour partie fusionner avec elles.»

La prédiction de Wilson s'est réalisée de manière éclatante avec le livre récent de Jared Diamond, Guns, Germs and Steel; I'auteur y étudie l'influence de l'environnement sur l'histoire des différentes sociétés humaines dans la perspective des sciences du vivant, et en particulier la théorie évolutionniste. Voici ce qu'il écrit:

L'idée que la biogéographie de l'environnement et la biogéographie ont influencé le développement de la société est naturellement une vieille idée. De nos jours, cependant, les historiens ne s'en font pas grand cas: ils la jugent fausse ou simpliste, la caricaturent ou la rejettent comme une forme de déterminisme par le milieu [...]. Or, à l'évidence, la géographie a eu quelque effet sur l'histoire: toute la question est de savoir à quel point et dans quelle mesure la géographie peut expliquer la configuration générale de l'histoire.

Le temps est venu de porter un regard neuf sur ces questions, en raison des données nouvelles venues de disciplines scientifiques apparemment éloignées de l'histoire humaine. Ces disciplines sont nombreuses: avant tout la génétique, la biologie moléculaire, la biogéographie et appliquées aux cultures et à leurs ancêtres sauvages; les mêmes disciplines et l'écologie des comportements appliquées aux animaux domestiques et à leurs ancêtres sauvages; la biologie moléculaire des germes humains et des germes apparentés des animaux; l'épidémiologie des maladies humaines; la génétique humaine; la linguistique; les études archéologiques sur tous 
les continents et des grandes îles; et l'histoire de la technologie, l'écriture et l'organisation politique ${ }^{17}$.

La formation pluridisciplinaire de Diamond est un atout, du point de vue de cette difficile «synthèse" vers laquelle il tend - et qui ressemble comme deux gouttes d'eau à la «convergence» théorisée par Wilson. Tout en s'excusant par avance pour son «optimisme impénitent », Wilson n'en réaffirme pas moins sa confiance enthousiaste dans la science, qu'il appelle «de toutes les métaphysiques, la plus audacieuse pour notre temps". Il voit en elle le moteur de la convergence des savoirs. Les chapitres du livre de Wilson consacrés aux arts (et à la critique artistique) d'une part, à l'éthique et à la religion d'autre part, attestent de la forte séduction qu'exercent sur lui les humanités. Ils devraient intéresser les littéraires, bien au-delà du cercle des chercheurs travaillant sur les relations entre textes et environnement. En vérité, des lors que la convergence des savoirs, telle qu'il la définit, a partie liée avec notre capacité à relever des défis planétaires comme la surpopulation, les menacés sur la biodiversité, ou la destruction d'écosystèmes entiers, tout lecteur devrait se sentir concerné.

Pour autant, je kuis prêt à reconnaître, après Snow, Huxley, et alii, que la science qui se prête le mieux à un rapprochement avec l'analyse des textes (dans l'écocritique), à savoir la biologie, est aussi la science la moins exacte, du moins celle qui occasionne le plus de conflits d'interprétations. Les réactions pour le moins mitigées à l'émergence de la sociobiologie, qui rappellent l'accueil non moins contrasté fait autrefois aux théories darwiniennes, illustrent le caractère conflictuel de la biologie; ce genre de réception est une incitation permanente, non seulement à affiner les résultats, mais à accepter d'en débattre avec un public informé. En même temps, nous ne pouvons pas faire comme si, en biologie, on partait à chaque fois de zéro. Que la nature soit une construction sociale, d'accord; mais cet énoncé, à lui tout seul, ne permet pas une meilleure compréhension scientifique de notre situation dans la biosphère. Toutes les approches «culturelles" de la nature ne se valent pas au plan scientifique. Nous en sommes tellement à célébrer la diversité des cultures que nous avons fini par oublier à quel point les humains se ressemblent. Argumenter pour savoir si la nature humaine

17. Jared Diamond, Guns, Germs, and Steel, New York, 1997, p. 25-26. 
est bonne ou mauvaise par essence est une chose, mais - comme le dit Mary Midgley - "c'est autre chose de nier, comme le veut la doxa actuelle, qu'il y ait quelque nature humaine que ce soit $^{18}$ ".

Le philosophe Alexander Rosenberg est le dernier en date à nous avoir rappelé le risque de dérapage des théories biologiques. Tout en rappelant, après Snow et Huxley, que les résultats de la biologie sont beaucoup plus difficiles à vérifier que ceux d'autres sciences comme la physique ou la chimie, Rosenberg pense qu'elle n'en constitue pas moins « une extraordinaire batterie d'instruments utiles pour repenser notre interaction avec le biocosmos ${ }^{19}$ ». Étant donné qu'il s'agit là tout à la fois de la question déterminante pour le siècle à venir, et de la raison d'être de l'écocritique, la biologie va se voir attribuer un rôle de plus en plus important.

Je voudrais, en conclusion, m'appuyer sur l'appel lancé par Snow aux intellectuels venus de toutes les disciplines mais ayant en commun d'être engagés dans un dialogue constructif avec les sciences. C'est à la fin de son livre Les Deux Cultures (1964). Ce manifeste pour l'émergence d'une «troisième culture" peut servir d'inspiration à tous les humanists, dont je souhaite qu'ils soient de plus en plus nombreux à utiliser les concepts de l'écologie dans l'écriture, la lecture, l'enseignement et la critique des textes littéraires.

Le problème majeur auquel est confrontée l'écocritique est l'inertie du champ de la critique littéraire contemporaine. William Howarth a judicieusement fait remarquer que les chercheurs de toutes les disciplines ont tendance à résister aux nouvelles approches; tout en faisant mine officiellement d'approuver l'innovation, ils ne sortent guère des sentiers battus. «Les théoriciens sont prompts à regarder de haut l'écocritique, accusée d'avoir des problématiques assez pauvres - comprenez, qui ne font pas écho aux idéologies à la mode. Certes l'éthique politique est bienvenue, mais pas si elle traite en priorité de sujets non humains, comme le paysage, les animaux, ou l'enfouissement des déchets ${ }^{20}$.» Cette résistance découle du point de vue conventionnel selon lequel les humanists étudient l'humanité, alors que des sujets

18. Mary Midgley, Beast and Man, Ithaca, Cornell University Press, 1978, p. 68.

19. Alexander Rosenberg, Instrumental Biology or The Disunity of Science, Chicago, University of Chicago Press, 1994. p. 1.

20. William Howarth, « Some Principles of Ecocriticism», dans Cheryll Glotfelty et Harold Fromm. The Ecocriticism Reader. Athens, The University of Georgia Press, 1996, p. 77. 
comme l'environnement sont du domaine des scientifiques; Neil Evernden fait observer, non sans ironie, qu'étudier l'humanité en la coupant de son environnement est un exemple parfait du réductionnisme que les premiers aiment tant à critiquer chez les seconds. "Je suis frappé à quel point l'idée même que l'homme doive s'engager envers d'autres créatures que lui-même, qu'il est tenu à certains impératifs biologiques, est considéré dans certains milieux comme un affront fait à l'humanité», écrit Evernden. Les humanists devraient au contraire être les premiers concernés par les questions d'environnement, poursuit-il, parce qu'elles sont d'ordre éthique, et que «l'éthique et l'art sont les deux faces d'une même médaille. L'environnement sans l'esthétique, c'est de l'aménagement du territoire local ${ }^{21}$ ».

L'establishment des études littéraires n'est pas le seul à refuser de lier la condition humaine à des contextes plus larges et non humains; il en va de même pour de nombreux écrivains contemporains, "Pourquoi la fiction américaine de ces dernières années est-elle si stérile?» se demande Scott Russell Sanders. Contre la tradition dominante de la littérature américaine, consistant à situer l'action dans une vaste nature englobante, «le monde littéraire grouille aujourd'hui de magiciens de plus en plus nombreux à nous concocter des potions de plus en plus insipides... Une fiction peut rendre parfaitement l'écume des jours; mais il y a quelque chose de profondément artificiel et presque pathologique dans le fait de jamais s'aventurer au-delà du monde infrahumain $^{22}$ ». Les problèmes sans précédents auxquels nous sommes confrontés à l'aube du XXI ${ }^{\mathrm{e}}$ siècle, qu'il s'agisse de surpopulation, de pollution, de changement climatique, ou d'atteintes à la biodiversité, semblent valider le jugement de Sanders. Nous sommes - Jared Diamond nous le rappelle - la première génération dans toute l'histoire humaine à devoir nous poser la question de la survie de l'espèce: les générations à venir pourront elles continuer d'habiter cette planète ${ }^{23}$ ?

Dans le même temps, il existe des signes positifs d'évolution des mentalités: écrivains et critiques commencent à comprendre qu'une littérature contemporaine qui prétend faire face à la réalité mondiale se doit d'avoir une composante environnementale. L'époque semble favo-

21. Neil Evernden, «Beyond Ecology: Self, Place, and the Pathetic Fallacy », ibidem, p. 102-103.

22. Scott Russell Sanders, «Speaking a Word For Nature », ibidem, p. 182, 194.

23. Jared Diamond, The Rise and Fall of the Third Chimpanzee, London, 1991, p. 313. 
rable à l'épanouissement d'une écocritique sérieuse au plan scientifique, qu'il s'agisse de pollution, de défense du terroir (bioregionalism), ou de la vie des animaux. Prenons White Noise, qui valut à Don DeLillo le National Book Award en 1985: Sanders y voit l'exemple parfait du roman enlisé dans le monde infrahumain; je suis au contraire frappé, comme Frank Lentricchia, par la nouveauté de la vision environnementale de DeLillo, qui signe là un «roman écologique précurseur de la conscience écologique ${ }^{24}$ ». Jack Gladney le narrateur, et sa famille, sont directement affectés par les retombées d'une catastrophe chimique, alors même que l'alerte officielle ne parle que de «pollution atmosphérique». Au début, Gladney se rebelle: «Je suis quand même un professeur, à la tête d'un département de mon college. Je ne vais quand même pas m'enfuir à cause d'une pollution atmosphérique. Ça, c'est bon pour les prolos du comté, qui vivent dans des mobile homes et travaillent dans les conserveries de poissons ${ }^{25}$.» Mais il doit s'enfuir comme les autres. La situation dans le livre est tout a fait plausible; à Bhopal comme à Tchernobyl, des «catastrophes écologiques" similaires venaient de tuer ou de contaminer mortellement des milliers d'individus. Presque au moment de la parution du roman, on frôla le désastre avec Three Mile Island.

Bref, le monde naturel, qui pour Sanders est absent de White Noise est en fait bien là, après tout, comme une sorte de présence sublimée -l'équivalent de ces non-dits auxquels Willa Cather attribue de si grands pouvoirs, ou de l'iceberg dont les neuf dixièmes sont immergés dans la nouvelle de Hemingway. La nature s'impose partout dans le roman, même dans les produits littéraires standard pour supermarchés, où tout se veut lisse et épuré. C'est bien d'elle qu'il s'agit, quand nous regardons de spectaculaires couchers de soleil en technicolor dus aux rejets de gaz toxiques, ou quand le vent lourd qui transporte les toxines mortelles renvoie les principaux personnages à la crainte obsédante de la mort, bien loin du fantasme postmoderne du corps postnaturel et postmortel. Le suréquipement technologique bruyant qui entoure les personnages de White Noise, "câblés" aux différentes chaînes TV - «Santé», «Météo», «News», «Nature»- fait un contrepoint ironique au monde naturel, qui exerce une sorte de veille silencieuse, en

24. « Introduction ", dans Frank Lentricchia (dir.), New Essays on White Noise, Cambridge, 1991, p. 7. 25. Don DeLillo, White Noise, New York, 1985, p. 117. 
marge de leurs vies artificielles et oublieuses de la bénédiction traditionnelle de l'Amérique: la capacité de s'évader et de se ressourcer à la campagne.

Leslie Marmon Silko aborde également les préoccupations environnementales à travers une fiction mémorable, Ceremony (1977). C'est l'histoire d'un individu qui ressent les menaces de la technologie moderne sur les paysages comme un empoisonnement de son être spirituel; c'est dans les vestiges de son patrimoine amérindien qu'il découvre la promesse d'une guérison de sa terre et de son peuple. Dans les deux romans que nous venons de citer, la technologie au service du seul profit est la cible d'une critique écologique. John Updike fait entendre la même musique, même si c'est en sourdine, dans le dernier épisode des aventures de Rabbit Angstrom, Rabbit at Rest (1990). On a parlé à ce sujet de "fiction post-nature ${ }^{26}$ »; Rabbit at Rest traite d'une Amérique déracinée, en proie à l'usure et à la toxicité. Le lyrisme que l'on trouve habituellement chez Updike y est comme pris au piège, ce piège de la spoliation et de la marchandisation de la nature qui a inspiré tant d'écrits pro-environnement ${ }^{27}$.

Pendant plus de deux mille ans, de Théocrite et Virgile en passant par la Renaissance, le romantisme et la période victorienne, et jusqu'à une période récente, la tradition vénérable de la pastorale fut l'objet et la raison d'être de ce qu'on pourrait appeler une éco-critique d'avant la chute. Récemment, toutefois, cette approche traditionaliste, imprégnée de nostalgie des origines, a radicalement changé sous la pression des changements écologiques: il n'est plus possible de continuer à se bercer d'illusions réconfortantes au contact d'une nature stylisée, qui serait -comme dans la pastorale- le lieu de la réconciliation de l'homme avec son milieu ${ }^{28}$. La dégradation et la toxicité de l'environnement sont des thèmes de plus en plus présents, non seulement dans les romans évoqués plus haut, mais aussi dans la science-fiction et dans les short

26. Cynthia Deitering, «The Postnatural Novel: Toxic Consciousness in Fiction of the 1980s », dans Glotfelty and Fromm, Ecocriticism Reader, p. 199-200.

27. Sur le traitement de la nature dans les premières fictions d'Updike, voir Larry E. Taylor, Pastoral and Anti-Pastoral Patterns in John Updike's Fiction, Carbondale, Southern Illinois University Press, 1971.

28. Voir Glen A. Love, «Et in Arcadia Ego: Pastoral Theory Meets Ecocriticism n, Western American Literature, vol. 23, 1992, p. 195-207. 
novels $^{29}$, où ils s'accompagnent, fait notable, de considérations de justice sociale - puisque les premières victimes de cette dégradation sont les plus démunis.

La montée en puissance de technologies de plus en plus agressives a également revigoré le souci du territoire, de la région, ou de la «biorégion" comme on dit maintenant, dans toutes leurs dimensions, humaines et extrahumaines. «De tous les besoins de l'âme humaine, disait Simone Weil, il n'en est pas de plus vital et de moins reconnu que le besoin d'enracinement ${ }^{30}$. " Force est de constater que de plus en plus d'essayistes et auteurs de fiction analysent ce besoin, dans des livres fortement situés dans un terroir ${ }^{31}$. Certes, cette efflorescence ne saurait complètement surprendre, eu égard à la riche tradition littéraire nordaméricaine dans ce domaine, de Bartram et Cooper, à John Muir, Mary Austin, et Aldo Leopold, en passant par Thoreau.

Ce qui est plus surprenant, c'est le nombre de livres et d'articles sur ces thématiques écrits par des universitaires venus de disciplines aussi différentes que la phénoménologie, la géographie, l'anthropologie, I'histoire, et les sciences dures ${ }^{32}$. Ces travaux ont en commun d'insister sur l'importance, et l'intérêt, du lien entre l'homme et son milieu. Nous assistons donc à l'émergence impressionnante d'une nouvelle science du territoire, qui ne saurait laisser les littéraires indifférents: jusqu'à présent, malheureusement, bien rares sont ceux qui ont franchi la fameuse «barrière» entre les sciences et les humanités; la plupart du temps, les «littéraires" s'en tiennent à un usage métaphorique du langage, sans répondre à l'appel de l'interdisciplinarité ${ }^{33}$.

29. On a pu récemment repérer cette tendance chez des auteurs comme Ursula K. Le Guin, Kurt Vonnegut, Rick DeMarinis, Rudolfo Anaya, Edward Allen, John Edgar Wideman, et T. Coraghessan Boyle.

30. Cité dans Kirkpatrick Sale, Dwellers in the Land, San Francisco, 1985, p. 47.

31. Parmi ces auteurs, on peut citer Wallace Stegner, Wendell Berry, Gary Snyder, Annie Dillard, Edward Abbey, Joan Didion, Barry Lopez, Rick Bass, et Terry Tempest Williams, pour en nommer seulement quelque-uns.

32. Voir en particulier Maurice Merleau-Ponty et Edward S. Casey; Yi-Fu Tuan, Edward Relph, et Douglas C. Pocock; Keith Basso; William Cronon et Donald Wooster; James Lovelock, Paul Shepard, Jared Diamond, et Gary Paul Nabhan.

33. Des universitaires spécialisés en sciences humaines ont mené des recherches en ce sens. Voir notamment Michael Kowalewski, «Bioregional Perspectives in American Literature», dans David Jordan (dir.), Regionalism Reconsidered, New York, 1994, p. 29-46; Lawrence Buell, « Place n, dans The Environmental Imagination, op. cit., p. 252-279; et Peter Blakemore, Writing Home, thèse de doctorat non publiée, University of Oregon, 1998. 
Il convient, pour finir, de faire état d'un sujet qui me semble particulièrement attractif pour les étudiants en écologie littéraire: il s'agit des rapports entre animaux humains et non humains - une longue histoire, qui demande à être revisitée. Darwin le premier nous força à reconsidérer notre rapport à l'animal. Les recherches et découvertes récentes nous invitent à approfondir, et à raffiner, notre approche de l'animalsujet, loin des catégories simplistes d'autrefois. Que vaut le concept de frontière entre les espèces quand nous apprenons, par exemple, que nous partageons $98 \%$ de nos caractéristiques génétiques avec les chimpanzés - une proximité telle qu'elle ne peut manquer de fasciner?

Dans le même temps que notre perception traditionnelle de l'autre animal est rendue problématique par les acquis de la recherche, les animaux tiennent une plus grande place dans nos vies et dans nos pensées. Ceci apparaît clairement dans une étude récente sur les victimes animales dans la littérature moderne: la thèse défendue est que les animaux « remettent en question les théories qui tendent à les nier dans leur spécificité. Cette résistance des animaux au contrôle par les humains (enculturation) n'est pas sans conséquence pour les questions difficiles et essentielles que se pose la littérature ${ }^{34} »$.

La vie des animaux est un sujet qu'explorent également, avec des résultats étonnants, des poètes comme Denise Levertov, Mary Oliver, John Haines, et bien d'autres. La création contemporaine mérite toute notre attention. Une nouvelle vague de poètes naturalistes est apparue: ils sont de leur temps, c'est-à-dire écologistes, et ils ne voient plus la nature comme permanente et inépuisable, mais comme vulnérable et créant de nouvelles et lourdes responsabilités - lourdes, car notre identité culturelle est en jeu ${ }^{35}$. L'esprit de cette nouvelle poésie, qui consiste à réapprendre notre relation à la nature, est exprimé par Wendell Berry quand il estime que «la base implicite de tout, c'est l'humilité, la réti-

\footnotetext{
34. Marian Scholtmeijer, Animal Victims in Modern Fiction, Toronto, 1993, p. 8 [dorénavant noté AV]. L'esprit des animaux non humains a attiré l'attention d'auteurs de récits non fictionnels informés par la biologie, tels Lopez, Peter Matthiessen, David Quammen, et Vicki Hearne, et de scientifiques comme Shepard, Wilson, Richard Nelson, Jane Goodall, et Stephen Jay Gould.

35. Parmi les récentes anthologies représentatives du mouvement de poésie naturaliste, on peut citer Robert Bly (dir.), News of the Universe, San Francisco, 1980; Christopher Merrill (dir.), The Forgotten Language, Salt Lake City, 1991 ; et Robert Pack and Jay Parini (dir.), Poems for a Small Planet, Hanover, 1993. Mentionnons également quelques ouvrages critiques sur la nature et la poésie, tels John Elder, Imagining the Earth, Urbana, 1985; et Andrew Elkins, The Great Poem of the Earth, Moscow, Id., 1997.
} 
cence à imposer un changement à l'ordre des choses, la volonté d'établir avec le monde des rapports d'élève et de serviteur, le souhait d'être inclus dans l'ordre naturel plutôt que de "conquérir la nature", le désir de découvrir les formes naturelles plutôt que de créer de nouvelles formes qui seraient humaines ${ }^{36} »$. Alors que l'environnement est sous pression comme jamais, la prise de conscience écologique contient la promesse de nouveaux poètes, relevant le double défi de la bioresponsablité et de l'innovation linguistique.

Évoquer la présence des animaux, c'est faire surgir toute l'histoire de leur représentation, ce qui me fait penser à une autre fonction importante de l'écocritique: réexaminer et réinterpréter les représentations de la nature dans les œuvres canoniques du passé. C'est ainsi que Joseph Meeker s'est livré à une relecture de la Divine Comédie de Dante et du Hamlet de Shakespeare (The Comedy of Survival); tandis que Jonathan Bate et Karl Kroeber revisitait les poètes romantiques anglais, Louise Westling appliquait la grille écoféministe aux classiques de la fiction américaine (The Green Breast of the New World). Comme je l'ai déjà indiqué, Moby Dick constitue une formidable opportunité pour l'écocritique appliquée à un classique. Les chapitres sur les baleines qui traditionnellement ont plongé non seulement les critiques, mais aussi les lecteurs, dans un certain embarras, peuvent constituer une intéressante entrée en matière. Sans remettre en cause l'immense complexité du roman de Melville, et de la multiplicité des points de vue dans Moby Dick, je pense que de nouvelles lectures, explorant ce que mon collègue David C. Morris appelle «l'être-baleine (whaleness) de la Baleine», ajouteraient encore à cette œuvre en révélant des niveaux de complexité ignorés $^{37}$.

Du point de vue évolutionniste et biologique, Moby Dick est peutêtre l'animal qui a la présence la plus forte de toute la littérature. De même, le narrateur qui interprète pour nous ses mouvements, Ismaël, peut être considéré non seulement comme un survivant, mais comme une étape dans le processus évolutionniste, au sens que Meeker donne à cette notion dans The Comedy of Survival. Ismaël incarne les qualités

36. Cité dans Merrill, The Forgotten Language, p. xvill.

37. On peut trouver des lectures critiques récentes de ce roman, envisagé du point de vue de l'étude des cétacés dans Robert Zoellner, The Salt-Sea Mastodon, Berkeley, 1973; Robert M. Greenberg. Splintered Worlds, Boston, 1993, p. 82-118; et Joseph Andriano, « Brother to Dragons: Race and Evolution in Moby Dick», American Transcendental Quarterly, vol. 10, 1996, p. 141-53. 
essentielles pour s'adapter et s'intégrer à un milieu: sa capacité à passer des compromis, à faire la paix, le rendent plus endurant qu'Achab, qui est le dernier de son espèce; Achab, dont la rage a quelque chose de tragique, car son choix de faire de la nature sa propre malédiction ne peut qu'amener un double désastre, personnel et écologique. Huit ans avant l'œuvre monumentale de Darwin, Moby Dick révèle l'intuition (implicite) de Melville que «l'humanité ne pourra à l'avenir détruire Moby Dick et ses semblables sans s'infliger à elle-même de réels dommage psychiques et concrets» $[A V$, p. 51$]$.

Repenser le canon dans ces termes ouvre de nombreuses possibilités. Un siècle d'études sur le comportement et le capital génétique des humains et des animaux a profondément modifié notre conception de la nature humaine, et notre façon de penser le rapport nature $v s$ culture. Le première notion qui me vient à l'esprit pour reconstruire la littérature est le naturalisme, qui n'a pas bonne presse à cause de son association avec le darwinisme social - une expression qui mériterait un réexamen. Le travail des écrivains dits "naturalistes ${ }^{38}$ » devrait susciter de nouvelles interprétations de la part de l'écocritique, que ce soit dans une perspective évolutionniste ou écomarxiste ${ }^{39}$. De même, le temps est venu de relire les œuvres «réalistes»: l'expérience nous montre chaque jour un peu plus que feindre d'ignorer la réalité ne la fait pas disparaître ${ }^{40}$.

Les implications sociales des progrès en biologie constituent l'un des plus grands bouleversements intellectuels de notre temps. Cela devrait suffire pour susciter l'attention et soutenir l'intérêt de tous ceux qui se sentent concernés par l'avenir de l'humanité sur cette planète. L'écocritique sérieuse, celle qui prend la peine de s'informer scientifiquement, fournit l'occasion de redynamiser l'enseignement et l'étude de la littérature; elle peut aider à réorienter la critique littéraire, pour lui redonner un rôle majeur sur le plan politique et social.

Traduit de l'américain par Laurent Ferri avec l'aimable autorisation de l'auteur et de la revue New Literary History.

C 1999, Glen Love.

\footnotetext{
38. Par exemple, Jack London, Stephen Crane, Frank Norris, Edith Wharton, Theodore Dreiser, John Dos Passos, Djuna Barnes, Richard Wright, James T. Farrell, Ernest Hemingway, et John Steinbeck. 39. Susan F. Beegel et alii (dir.), Steinbeck and the Environment, Tuscaloosa, 1997, illustre de manière impressionnante les possibilités offertes par le croisement des disciplines.

40. Une lecture particulièrement novatrice de l'influence de l'ouvrage de Darwin, The Descent of Man and Selection in Relation to Sex, figure dans Bert Bender's, The Descent of Love, Philadelphia, 1996.
} 